\title{
MR Compatibility of Mechatronic Devices: Design Criteria
}

\author{
Kiyoyuki Chinzei $^{1}$, Ron Kikinis ${ }^{2}$, and Ferenc A. Jolesz ${ }^{2}$ \\ 1 Mechanical Engineering Laboratory, AIST, MITI \\ 1-2 Namiki, Tsukuba, 305-8564 Japan \\ chin@mel.go.jp \\ 2 Department of Radiology, Brigham and Women's Hospital \\ Francis St. 75, Boston, MA 02115, USA \\ \{kikinis, jolesz\}@bwh.harvard.edu
}

\begin{abstract}
Criteria to design MR (Magnetic Resonance) compatible mechatronic devices, e.g., surgical robots, are discussed. Some of critical interactions between MRI and mechatronic devices are discussed. Experimental results of the effects from several passive and active mechanical elements are demonstrated.

Some passive elements, e.g., ball screw and linear guide have point contacts therefore, they are required to be made of hard smooth surfaces. Both beryllium-copper and a new stainless steel were examined. Effects from ultrasonic motor were also examined. They didn't show any image shift. Significant degradation of signal noise ratio was not observed, too. Based on these results, we developed a five d.o.f. MR compatible surgical manipulator.
\end{abstract}

\section{Introduction}

MR compatibility has been a tough hurdle for device developers, in particular, for mechatronic developers who want to design robot. It is believed that the common standard mechanical parts cannot be used in MR environment because they usually contain ferromagnetic components. However, open configuration scanners have opened a door to the intraoperative use of MRI, where actuated parts have been used in some of the surgical equipment, e.g., respirator, etc [1].

Shellock intensively studied this subject and issued a guidebook of the compatibility to many medical devices [2]. Schenck defined magnetic MR compatibility and classified numerous materials [3]. GE Medical System disclosed its guideline in the homepage to design MR compatible devices for its intraoperative scanner [4]. It provides quite comprehensive and descriptive information about how developers should test the compatibility of their products.

Hynynen has developed MR guided focused ultrasound system [5]. It was actuated by ultrasonic (piezoelectric) motors. Masamune developed an MR compatible surgical manipulator, which also employed ultrasonic motors [6]. One of the authors has developed a few mechanical instruments which were intended to be used in MR environment. 
This paper will illustrate the criteria to design mechatronic devices to be MR compatible, assuming their use with open configuration scanners. This paper first reviews the MR compatibility, then summarizes any possible interaction between the mechatronic devices and the MR imaging. The effects caused by several metal samples and mechanical parts in the intraoperative MR scanner are demonstrated.

\section{MR Compatibility}

The most descriptive and practical definition of MR compatibility is found in [4]. It describes the experimental protocols to evaluate compatibility, which is introduced in this paper [4].

First [4] defines $M R$ environment as, the area of influence, in particular, inside the 5 Gauss line. 'Influence' can be more than magnetic, as discussed in Section 3. Then [4] states that a foreign device is $M R$ safe when it does not add risk to human or any equipment by placing it in the MR environment, however, it may effect imaging quality.

Finally, [4] defines $M R$ compatibility of a foreign device as;

- it is MR safe,

- its use in the MR environment does not affect imaging quality,

- it operates as designed in the MR environment.

In addition, [4] defines location and timing zones, where MR compatibility with respect to each zone should be stated. The zones are defined as followings:

Zone 1 device may remain in the image's region of interest and in contact with the patient during the surgical procedure and imaging.

Zone 2 device may remain in the imaging volume and in contact with the patient during the surgical procedure and imaging, but the device is not in the region of interest.

Zone 3 device is used within the imaging volume, but removed during imaging or when not in use.

Zone 4 device can be used in the magnet room during the surgical procedure if it is kept a distance of more than $1 \mathrm{~m}$ from the magnet center or outside the 200 Gauss line. ${ }^{1}$

\section{Interaction between MRI and Mechatronic Devices}

\subsection{Definition of Mechatronic Device}

In this paper, we refer to mechatronic devices, e.g., robot, as the composite of the mechanical and control (electric) parts. The mechanical part is composed from four elements: structural element, passive and active mechanical devices, and sensors. The control part can be outside the MR environment, therefore this paper is concerned with the behavior of the mechanical parts.

\footnotetext{
${ }^{1}$ The distance should be appropriately interpreted depending on the scanner.
} 


\subsection{Possible Interaction}

Here are various phenomena that can happen when a mechatronic device is placed adjacent to MRI scanner and is driven it during imaging.

Effect 1: Magnetic field affects mechanical devices. The strong static magnetic field can affect ferrous parts in the passive and active devices. This may result unexpected behaviors. For example, standard springs often do not function as expected inside zone 3.

Effect 2: RF pulse affects sensors. High-impedance sensors can induce the $\mathrm{RF}$ (radio frequency) pulse depending on the distance from and directivity of the RF coil. It is not easy to eliminate such induced signals. However, optics based sensors, e.g., optical encoders, can be free from this, if photo sensors and its amplifier are placed carefully.

Effect 3: Foreign objects affect magnetic field. The effect of ferromagnetic objects to the homogeneity of the magnetic field is obvious. In fact, even paramagnetic object can have an effect if it was conductive due to the eddy current in zones 1 to 2 .

Most of standard mechatronic devices are magnetically very incompatible.

Effect 4: Foreign objects affect RF probe. The RF probe is a receiver antenna and is tuned to the resonance frequency. Foreign objects that are dielectric or conductive, and are adjacent to the probe, typically in zones 1 to 2 , can affect the property of the antenna.

Effect 5: Wiring introduces noises. MR magnet room is an RF shield room. It cuts off electric noise from the outside and vice versa.

The wire to the device can act as an antenna radiating electric noise from the outside, regardless of the distance from the scanner. It significantly affects the image quality, in particular, its signal to noise ratio.

Effect 6: Foreign resonant objects affect gain controller. The gain controller of the signal receiver can be mistuned in the presence of a large source of resonance signal in zone 1 when the imaging object has weak signal. This can occur when the imaging object is small in volume and a hydraulic or water driven actuator is in zone 1 .

\section{Design Criteria}

\subsection{Device Design}

Structural Element. Many non-ferrous metals, ceramics, plastics, and composite materials are non-magnetic. Due to cost, strength and easiness of manufacturing, non-ferrous metals are the second best.

Austenitic stainless steels (300 series) are neither ferromagnetic, nor paramagnetic. Their magnetic susceptibility range from $10^{-1}$ to $10^{-3}$. Titanium and Aluminum are well known as MR compatible, but they are not ideally paramagnetic, hence they require some compromise if they were to be used in zones 1 to 2. Other materials are often too soft except ceramics, which are too hard and brittle. 
Passive Mechanical Devices. Gears, wire drive, cam, bearings, ball screw, linear guide, etc. are in this group. A variety of plastic parts are available. They are fine if rigidity is not required. Ceramic devices are expensive, brittle, and can be heavy. However, they are very hard and rigid. Ceramic bearings are widely available.

When frictionless is important, some devices, e.g., bearings, ball screws, etc., need the point contacts to transfer the load, hence they should have precise dimension and should be smooth as well as hard. Therefore, plastic parts are not suitable for this kind.

We examined ball screws made by paramagnetic metals, as will be described later.

Active Mechanical Devices. Actuators, clutches, and brakes fall into this category. Majority of these are electromagnetic device and practically impossible to use inside zone 3 .

Ultrasonic (piezoelectric) motor can be the substitute for electromagnetic motor $[7,8]$. Commercial non-magnetic products are available, however, they are electrically driven hence caution against any EMI (electromagnetic interference: discussed later) is required. Fluidic actuators, e.g., hydraulic or pneumatic actuators, can be also magnet free in principal. Transmission mechanisms, such as drive shafts, gears, and wires, allow the actuator to be placed away from MR scanner therefore compatibility criteria can be relaxed.

As far as actuators are concerned, there are other alternatives, however, it has been difficult to find good alternatives to electromagnetic clutches and brakes.

Sensors. Most of modern sensors are electric, which can emit noise to the imaging, and can receive signals from the RF pulse. The former can be improved by applying the general techniques against EMI, unless it is used in zones 1 to 2. Since the noise is a series of sharp pulses, standard EMI techniques, such as rejecting the common mode noise, are not effective, therefore it is a better practice to decouple the sensor and its wire from the RF coil.

As a solution the measured media should be placed far from the magnet. A case study was an optical encoder. Its photodiodes were located outside the magnet room and fiber optics were used to guide the signals to the optic sensors. This technique was shown to be effective.

\subsection{Electromagnetic Interference (EMI)}

EMI can occur even if the electric device is placed in zone 4. To minimize EMI, the following techniques must be considered:

- Wires and circuit should be properly shielded,

- Cables of appropriate size and impedance should be used,

- Twisted pair cables are recommended,

- Shield should be properly grounded. Large ground loop should be avoided, 
- Switching regulator and DC-DC converter, etc., should be avoided,

- Opto-isolation should be considered if necessary,

- Inline noise filters are often effective, etc.

\section{Alternative Devices and Simple Experiments}

Some alternative materials and devices were examined using the intraoperative MR scanner Signa/SP (GE Medical Systems, Milwaukee, WI, 0.5 tesla/60 cm, active shielding).

\subsection{Hard Paramagnetic Metal Chips in Zone 1}

The passive mechanical devices require precise dimensions, smooth and hard surface materials. As a substitute to steels, the following materials were tested: three austenitic stainless steels (type 304, 316, YHD50), and a beryllium-copper (BeA-25-HT; abbreviated as Be-Cu). YHD50 is a non-standardized new stainless steel (Hitachi Metals Ltd., Tokyo, Japan). Table 1 lists surface hardness and magnetic susceptibility of typical metals.

Table 1. Hardness and magnetic susceptibility of typical metals

\begin{tabular}{l|cc} 
& Hardness $(\mathrm{HB})$ & $\chi\left(\times 10^{-6}\right)$ \\
\hline Be-Cu & $300-380$ & 4 \\
\hline YHD50 & 420 & 1900 \\
\hline $\begin{array}{l}\text { Type 440C stainless } \\
\text { steel }\end{array}$ & 580 & $10^{9}$ \\
\hline $\begin{array}{l}\text { Type 316 stainless } \\
\text { steel }\end{array}$ & $<187$ & 9000 \\
\hline Al & $<150$ & $20.7[3]$ \\
\hline Ti & $>100$ & $182[3]$ \\
\hline
\end{tabular}

(Values from unpublished measurements by NSK Ltd. (Tokyo, Japan) otherwise indicated.)

Method. Five sample chips (stainless steels type 304, type 316, YHD50, YHD50 with surface hardening treatment, and Be-Cu) were prepared (Fig. 1). These had the identical cylindrical shapes of $20 \mathrm{~mm}$ in height and $20 \mathrm{~mm}$ in diameter. Each sample was put in $\mathrm{NiCl}_{2}$ solution so that its axis was aligned to the magnetic axis. We acquired a $2 \mathrm{D}$ scan that axially intersected the sample. Sequence was GRE, TE/TR: 30/150 msec, FOV: $260 \times 260 \mathrm{~mm}$, slice thickness: $4 \mathrm{~mm}$, bandwidth: $15.6 \mathrm{kHz}$, flip angle: 30 degrees. 
Result. Every metallic object produced a void (dark) shadow that had no resonance signal. The object also distorted the magnetic field around it. The larger the void was the larger distortion.

Beryllium-copper showed the smallest void. YHD50 was the second best followed by the stainless steel type 316, type 304 which was the worst (Fig. 2).

YHD50 after surface treatment showed larger void, which meant degradation of paramagnetism. It is known that austenitic stainless steel is so delicate that it can cause phase transfer to ferromagnetic by the cold working, e.g., bending [9]. MR compatibility should be assessed using the final products. Figure 3 shows the assessment of the final products of ball screws made of beryllium-copper and YHD50.

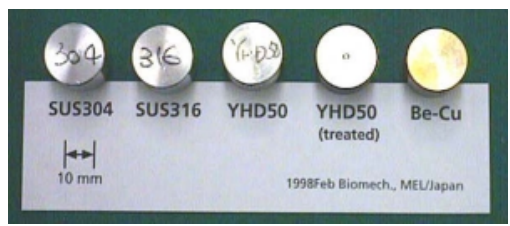

Fig. 1. Sample chips. From left, stainless steel type 304, type 316, YHD50, surface treated YHD50, and Be- $\mathrm{Cu}$.
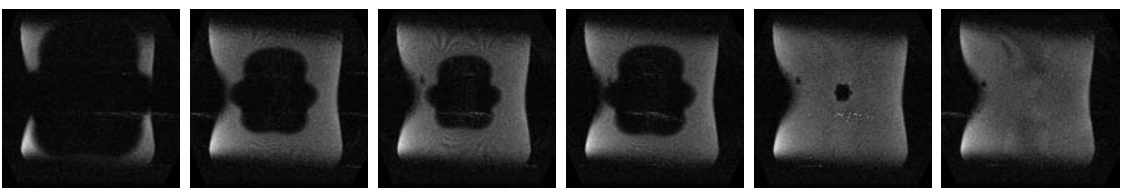

Fig. 2. Effect by metallic samples. From left, stainless steel type 304, type 316, YHD50, surface treated YHD50, Be-Cu, and the control, respectively. Void is caused by the distortion of the magnetic field.
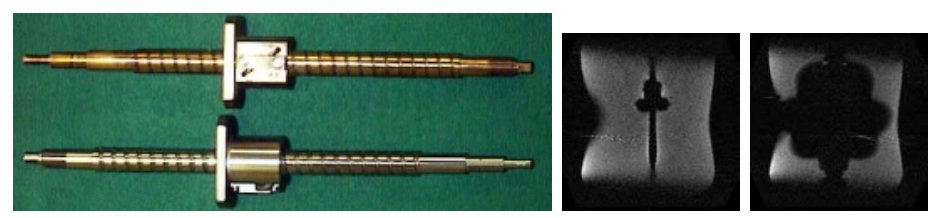

Fig. 3. Be-Cu (upper), YHD50 (lower) ball screws, and the effect by Be-Cu (center), YHD50 (right) ball screws.

\subsection{Ball Screws in Zones 2-3}

Two ball screws were examined; one made of YHD50 and another was made of beryllium-copper. 
Method. We put each ball screw in the scanner and observed the effect which appeared in the image by changing the distance between the screw and the center of imaging volume. The distances were selected at 240, 330 and $520 \mathrm{~mm}$. The ball screw was aligned parallel to the magnetic axis, or perpendicular to it. The control image was taken without the screw.

The imaging object was a spherical phantom of $\mathrm{CuSO}_{4}$ solution. The imaging sequence was SE, TE/TR: 17/400 msec, bandwidth: $15.6 \mathrm{kHz}$, FOV: $240 \times 240 \mathrm{~mm}$, Slice thickness: $5 \mathrm{~mm}$. As the phantom was kept immobilized, the images should stay identical.

Result. The obtained images looked identical. Thus, we list here the subtraction of the control image (Fig. 4).

To evaluate noise, Signal to noise ratio (SNR) was calculated:

$$
S N R=\text { Pcenter } / \text { SDcorner . }
$$

Where Pcenter is the mean value of $40 \times 40$ pixels area at the center of the image, SDcorner is the standard deviation of $40 \times 40$ pixels area at the lower right corner [4]. Table 2 lists the SNR values. The high SNR values correspond to high image quality. Note that, image shift and significant loss of SNR were not observed.

Table 2. Signal to noise ratio (SNR) of images with ball screws.

\begin{tabular}{l||lll} 
(distance from isocenter) & 240 & 330 & $520(\mathrm{~mm})$ \\
\hline Be-Cu ball screw (parallel) & 55.6 & 65.5 & 59.4 \\
Be-Cu ball screw (perpendicular) & - & 58.4 & 56.9 \\
YHD50 ball screw (parallel) & 57.5 & 56.6 & 62.9 \\
YHD50 ball screw (perpendicular) & - & 60.7 & 61.9 \\
\hline Control (no ball screw) & & 59.1
\end{tabular}

\subsection{Actuator: Ultrasonic Motor (USM) in Zones 2-3}

Method. An ultrasonic motor was placed in the scanner and the effect in imaging was observed. The motor was USM-60N1 (Shinsei Kogyo Corp., Tokyo, Japan; Fig. 5). The method described in Section 5.2 was repeated. The motor was driven at $75 \%$ of its maximum speed, or unplugged from its amplifier.

Result. Figure 6 shows the subtraction of the control, and Table 3 lists the SNR values.

Results show that there was neither image shift nor significant degradation of SNR values regardless of the motor being driven. 


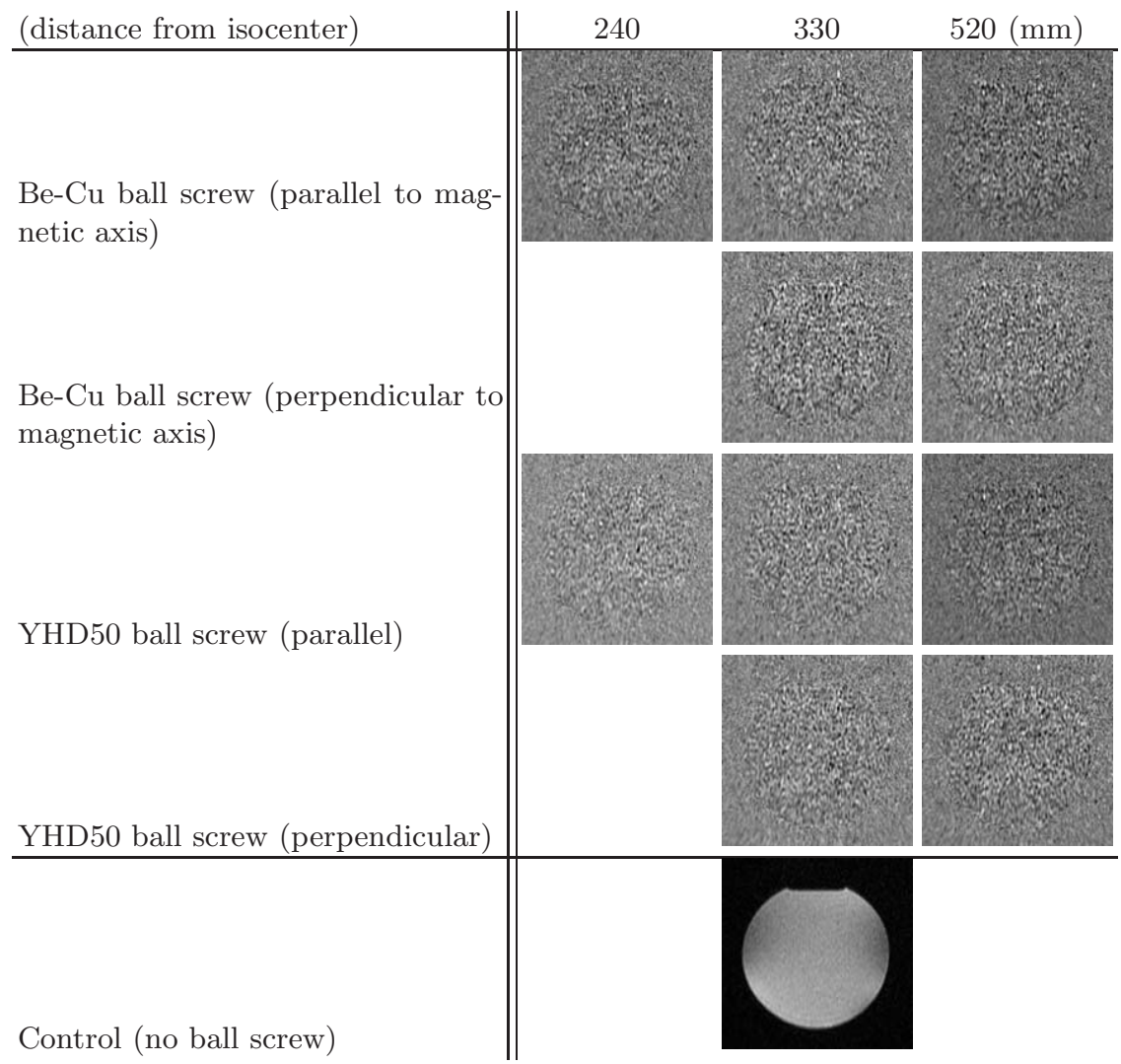

Fig. 4. Image shift by ball screws. Subtracted images are listed and no shift is found. (The ball screw could not be located perpendicular at $240 \mathrm{~mm}$ from the isocenter.)

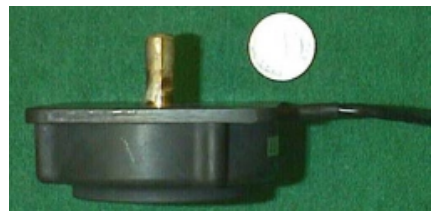

Fig. 5. Ultrasonic motor 


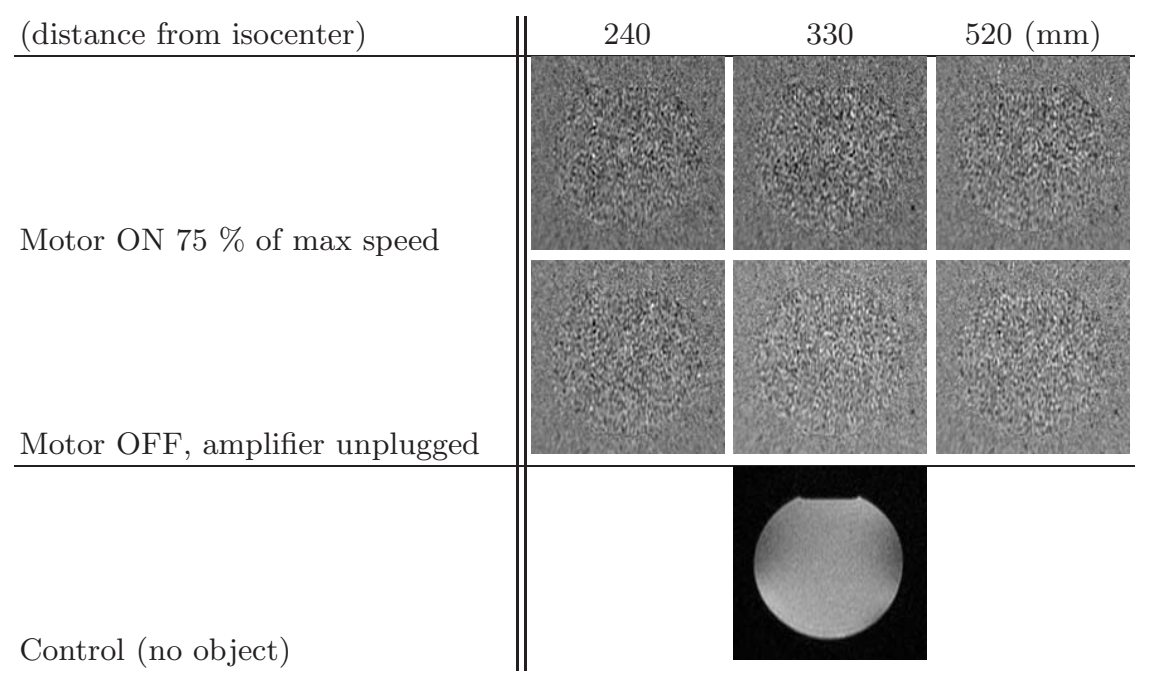

Fig. 6. Image shift by driving ultrasonic motor during imaging. Subtracted images are listed and no shift is found.

Table 3. Signal to noise ratio (SNR) of images with ultrasonic motor driving during imaging.

\begin{tabular}{l||lll} 
(distance from isocenter) & 240 & 330 & $520(\mathrm{~mm})$ \\
\hline Motor ON 75 \% of max speed & 54.4 & 64.0 & 55.5 \\
$\begin{array}{l}\text { Motor OFF, unplugged from ampli- } \\
\text { fier }\end{array}$ & 58.4 & 55.8 & 59.2 \\
\hline Control (no object) & & 59.1
\end{tabular}

\section{Discussion}

Possibility of MR Compatible Precise Mechanisms. Though these results are not meant to guarantee the acceptance of volumes or shapes of the foreign objects, they cast a spotlight to the possibility of MR compatible precision mechanisms. The results obtained from the study of the ball screw is encouraging to develop similar devices, such as linear guide.

Application: An MR Compatible Manipulator. The results have mainly been applied in the 5-axes surgical manipulator (Fig. 7). It is optimally designed for the intraoperative scanner and scheduled to be at the test stage shortly. It will be part of an image guided surgical assist system, which will integrate pre-operative planning and intra-operative MRI. 


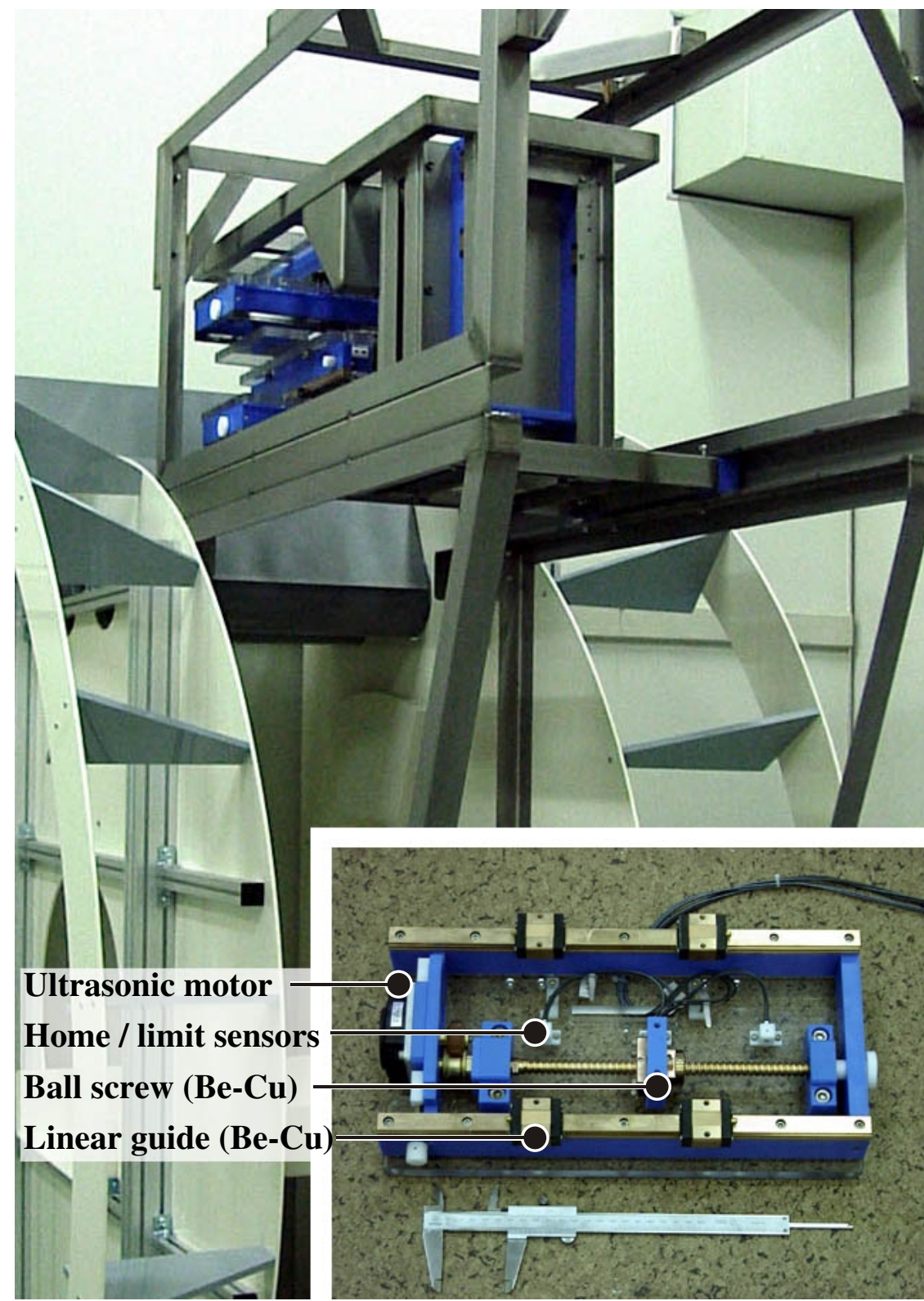

Fig. 7. Five d.o.f. MR compatible manipulator for the intraoperative MR scanner (main), and a linear motion table unit (bottom), made from MR compatible devices described in this paper. 
It was designed to guide surgical tools, such as biopsy needle, or pointing devices according to the preoperative planning. The manipulator consists of the main mechanical body and the tool holder hang from the former. The main body is placed above surgeon's head so that it does not block the surgeon accessing the patient. The tool holder contains neither delicate mechanical parts nor sensors, and is exchangeable and sterilizable.

It is a Cartesian type robot consisting of an X-Y-Z table with an additional horizontal X-Y table. Each table has a rigid arm and the ends of these arms, the tool holder, link together by two pivoted joints. Every table unit is composed from a ball screw and a pair of linear guides that are made of YHD50 or beryllium-copper. The actuators are the ultrasonic motors. All the sensors are optical and their circuits are placed outside the magnet room. Fiber optics are used for signal transmit.

\section{Conclusion}

The definition of MR compatibility was reviewed and the criteria to design mechatronic devices to be MR compatible were discussed.

Low susceptibility / high hardness stainless steel YHD50 and berylliumcopper (Be-Cu) as well as other standard stainless steels (type 304 and type 316) were examined in zone 1 of an intraoperative $\mathrm{MR}$ scanner. $\mathrm{Be}-\mathrm{Cu}$ was proven to be best performance to other materials, followed by YHD50. Ball screws made of these metals and an ultrasonic motor placed separately in zones 2-3 didn't show significant loss of SNR nor any image shift.

Though the result didn't guarantee the acceptable amount or shape of these objects, it encourages the possibility of the use of MR compatible components. Based on these results, an MR compatible surgical manipulator was approved for development.

\section{Acknowledgements}

The main part of this work has been funded by AIST, MITI, Japan. The application system described in Section 6 is bilaterally funded, in the US side by NSF ERL "Computer Integrated Surgical Systems and Technology" \#9731748.

\section{References}

1. Jolesz, F.A., Morrison, P.R., Koran, S.J., et.al.: Compatible instrumentation for intraoperative MRI: expanding resources. JMRI, 8(1) (1998) 8-11 1020

2. Shellock, F.G.: Pocket Guide to MR Procedures and Metallic Objects: Update 1998. Lippincott-Raven publishers, Philadelphia (1998) 1020

3. Schenck, J.F.: The role of magnetic susceptibility in magnetic resonance imaging: Magnetic field compatibility of the first and second kinds. Med. Phys., 23(6) (1996) 815-850 1020, 1024 
4. GE Medical Systems (ed): MR Safety and MR Compatibility: Test Guidelines for Signa $\mathrm{SP}^{\mathrm{TM}}$. Version 1.0, http://www.ge.com/medical/mr/iomri/safety.htm October (1997) 1020, 1021, 1026

5. Hynynen, K., Darkazanli, A., Unger, E., Schenck, J.F.: MRI-guided noninvasive ultrasound surgery. Med. Phys., 20 (1992) 107-116 1020

6. Masamune, K., Kobayashi, E., Masutani, Y., et.al.: Development of an MRI compatible Needle Insertion Manipulator for Stereotactic Neurosurgery. J Image Guided Surgery, 1 (1995) 242-248 1020

7. Sashida, T., Kenjo, T.: Introduction to Ultrasonic Motor (Chouonpa Motor Nyuumon; written in Japanese). Sogo Denshi Shuppan, Tokyo, (1991) 1023

8. Crivii, M., Jufer, M.: Piezoelectric ultrasonic motor - design and comparison. In proc Annu Symp Incremental Motion Control Syst Devices, 23 (1994) 35-40 1023

9. Bendel, L.P., Shellock, F.G., Steckel, M.: The effect of mechanical deformation on magnetic properties and MRI artifacts of type 304 and type 316L stainless steel. JMRI, 7(6) (1997) 1170-1173 1025 